\title{
AN ACOUSTIC RESPIROGRAPH. A METHOD FOR THE STUDY OF RESPIRATION THROUGH THE GRAPHIC RECORDING OF THE BREATH SOUNDS
}

\author{
BY SYDNEY MARGOLIN AND LAWRENCE S. KUBIE \\ (From the Neurological Institute of New York, and the Department of Neurology, College of \\ Physicians and Surgeons, Columbia University)
}

(Received for publication October 30, 1942)

During the course of an investigation of methods for producing hypnagogic states, it was noted that monotonous sounds were most efficacious when they occurred with a rhythmic frequency approximating that of the spontaneous respiratory rate of the subject. Consequently, a technique was developed for using the subject's breath sounds as the potent rhythmical stimulus. This instrument is described in previous communications (Kubie and Margolin $(1,2)$.

From the first, it was evident that the instrument provided the observers with an opportunity to study changes in the respiratory cycle under specially advantageous circumstances. The apparatus was an inertia-free system and introduced no obstruction either to the flow of air or to the respiratory movements. The subject was not necessarily aware that his respirations were under observation. Furthermore, the amplified breath sounds brought into clear relief each change that occurred from moment to moment in the course of the respiratory cycle. It was like listening to a heart through a stethoscope, instead of merely feeling the pulse at the wrist. For these reasons, and in order to use the method for more precise physiological studies and correlations, a direct writing recorder was devised to record the amplified breath sounds.

The construction of this recorder is based on the fact that the acoustic energy produced by the passage of air in the upper respiratory tract, is the resultant of a number of random frequencies ranging from 500 to 5,000 cycles per second. In order to eliminate all frequencies lying outside of this range, such as those produced by muscle contractions or by the movement of the skin under the microphone, and in order to minimize the influence of extraneous air-borne sounds, various commercial microphones were examined and experimental models made. One experimental mi- crophone was found to have both theoretical and actual advantages. This was a magnetic instrument with a rigid contact diaphragm, connected to a vibrating armature around which was a coil of No. 46 enamelled copper wire. However, since this type is not commercially available, a crystal microphone was used and found to be next in efficiency. ${ }^{1}$

When a direct contact microphone of this type is placed against the neck in the vicinity of the trachea, its electrical output is the resultant of alternating currents whose frequencies correspond with those of the breath sounds. These currents are led to a vacuum tube amplifier, the characteristics of which are such as to respond only to this range of frequencies, and to magnify them to a value sufficient to operate a direct writing galvanometer. The amplifier contains a rectifierand-filter system, which converts the random alternating currents into a voltage which varies directly with the magnitude of the respiratory acoustic energy, and at a rate which corresponds with the respiratory rate of the subject. This varying voltage in turn is led to a small power amplifier which drives the writing galvanometer. ${ }^{2}$ The ink-writer introduces no significant inertia into the system since it will respond with fidelity to a frequency of 100 per second, with negligible friction on the tape. Respiratory frequencies rarely attain a rate of fifty per minute. The record is inscribed on a moving strip of paper which is pulled by a constant speed electric motor. The rate can be varied by suitable gears from onehalf centimeter to three centimeters per second, the latter being the standard speed for electroencephalographic tape pullers. This makes possi-

1 This was the crystal contact microphone, marketed under the name of "Stethaphone" by the manufacturer, Shure Bros., Inc., Chicago, Ill.

2 The ink-writing magnetic galvanometer is made by the Electro-Physical Laboratories, New York, N. Y. 
ble a simultaneous recording and direct comparison of electroencephalograms and acoustic respirograms (Figs. 1 and 2).

This recording system has two outstanding advantages. The necessary amplification is carried out at high frequencies, where vacuum tube amplifiers are simple in construction and stable in operation. Secondly, the only connection between the subject and the apparatus is a small contact microphone. This makes it possible to obtain records without the subject's knowledge that his respiration is being studied, and under conditions which provide a minimum of restriction to his movements, and none to the moving current of air.

By the graphic representation of the sound which is produced by the movement of air currents in the upper respiratory tract, the respiratory cycle can be analyzed into several clearly recognizable phases.

At the onset of inspiration, there is a sudden rapid up-curve as the moving column of air gathers velocity and as the sound gathers volume. At some point on this curve, there is a levelling off to form something approximating a plateau, which in turn may be level, may continue slowly to rise, or may gradually fall. At the end of inspiration, there is a sharp drop back to the base line. Then comes a quiet lull during the interphase between inspiration and expiration, a phase which is lost to view in mechanical methods of registering the respiratory movements of the thorax or abdomen. This interphase terminates where the onset of expiration causes a second rapid rise in the curve, a second plateau as the expelled column of air attains a more or less constant velocity, and again a sharp drop at the end of expiration. Thus, there is an up-slope, an approximate plateau, and a down-slope for both the inspiratory and the expiratory phases of respiration, and two interphases, one between inspiration and expiration, and another between expiration and inspiration. The sharpness of these slopes, the inclinations of the plateaux, the relative durations of the various phases and interphases, and their smoothness or jerkiness, all give insight into variations in the respiratory function. Not only are gross irregularities of rhythm clearly revealed, but also many subtler patterns which elude ordinary inspection and such methods of

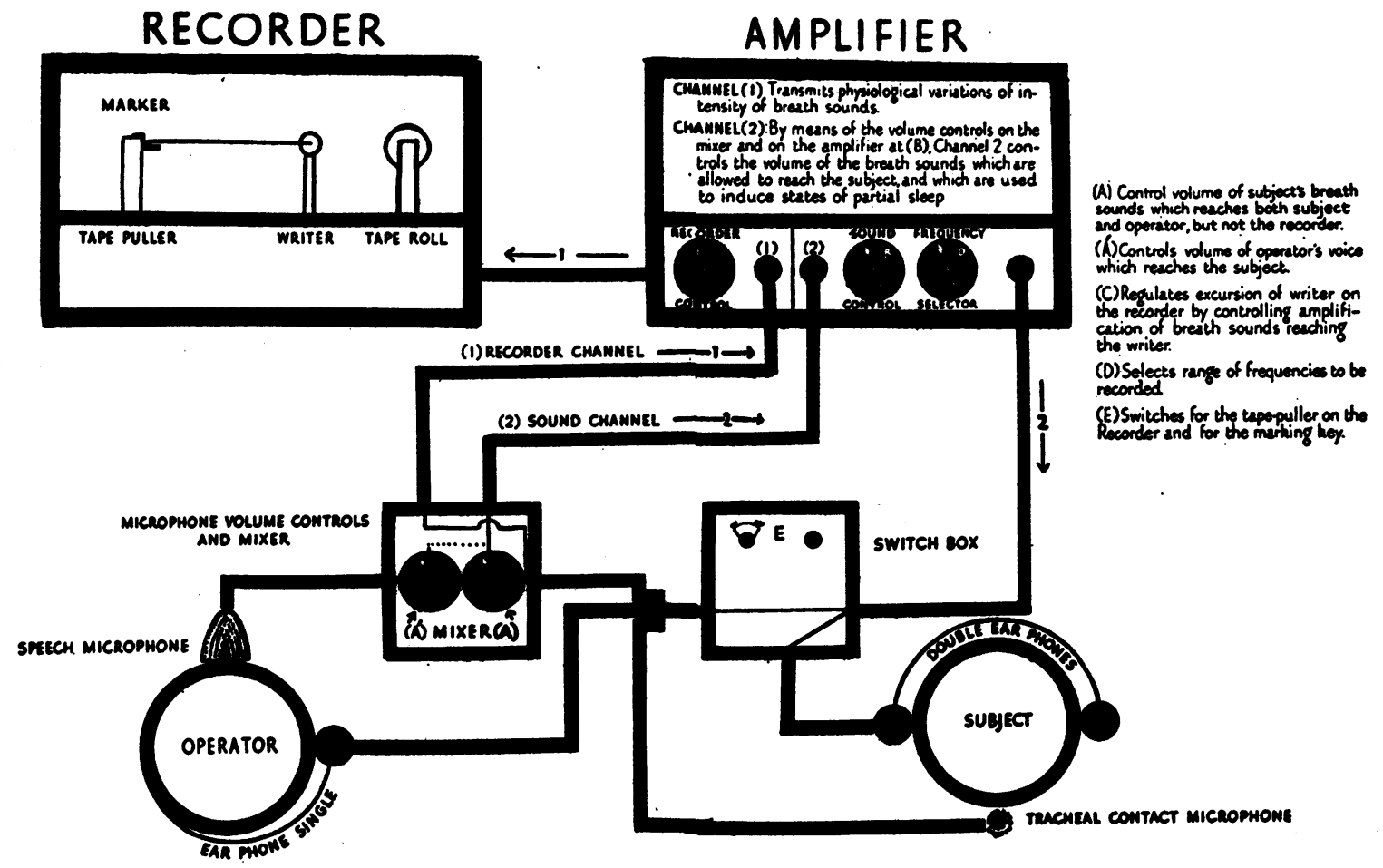

Fig. 1. Acoustic Respirograph 


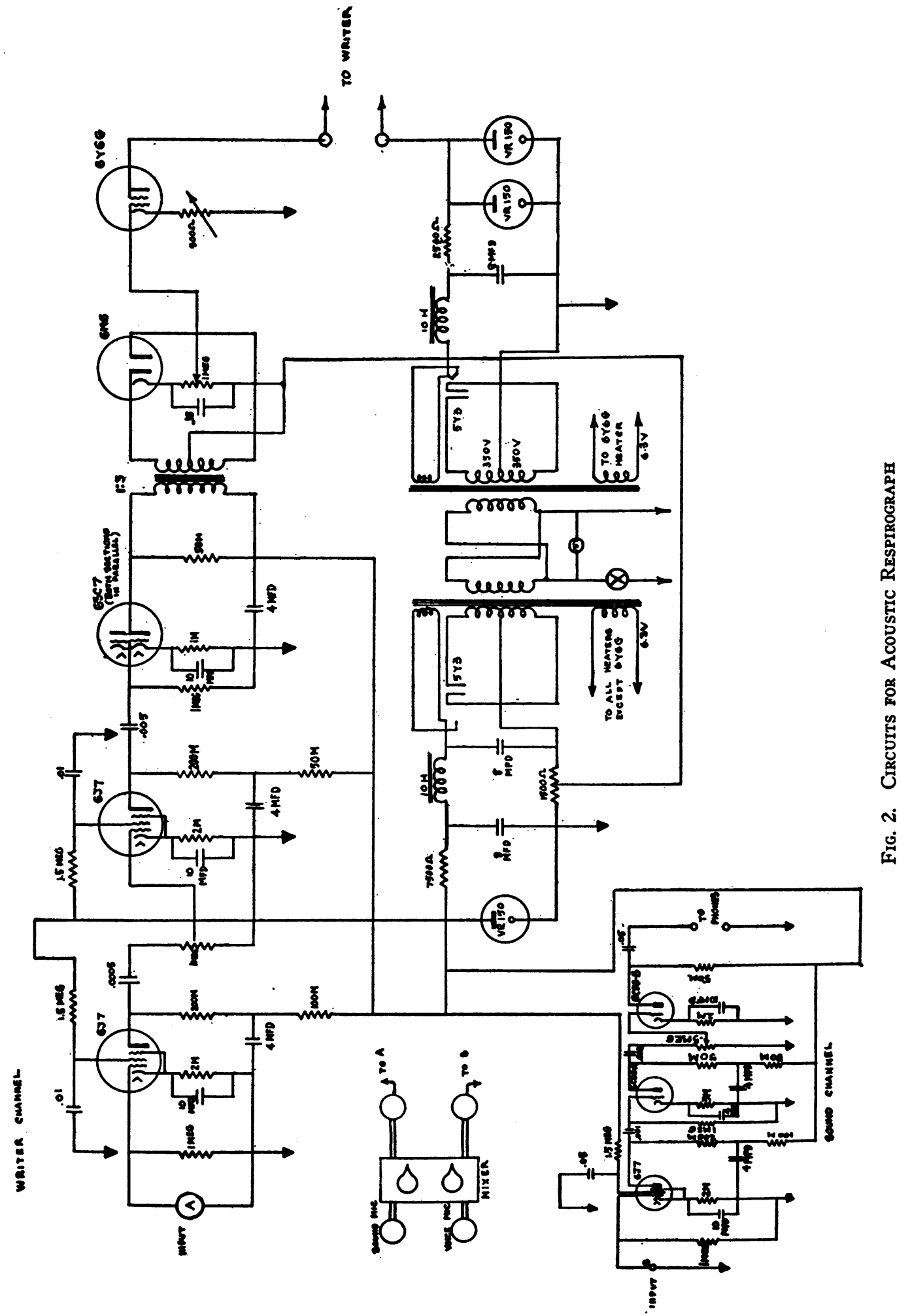



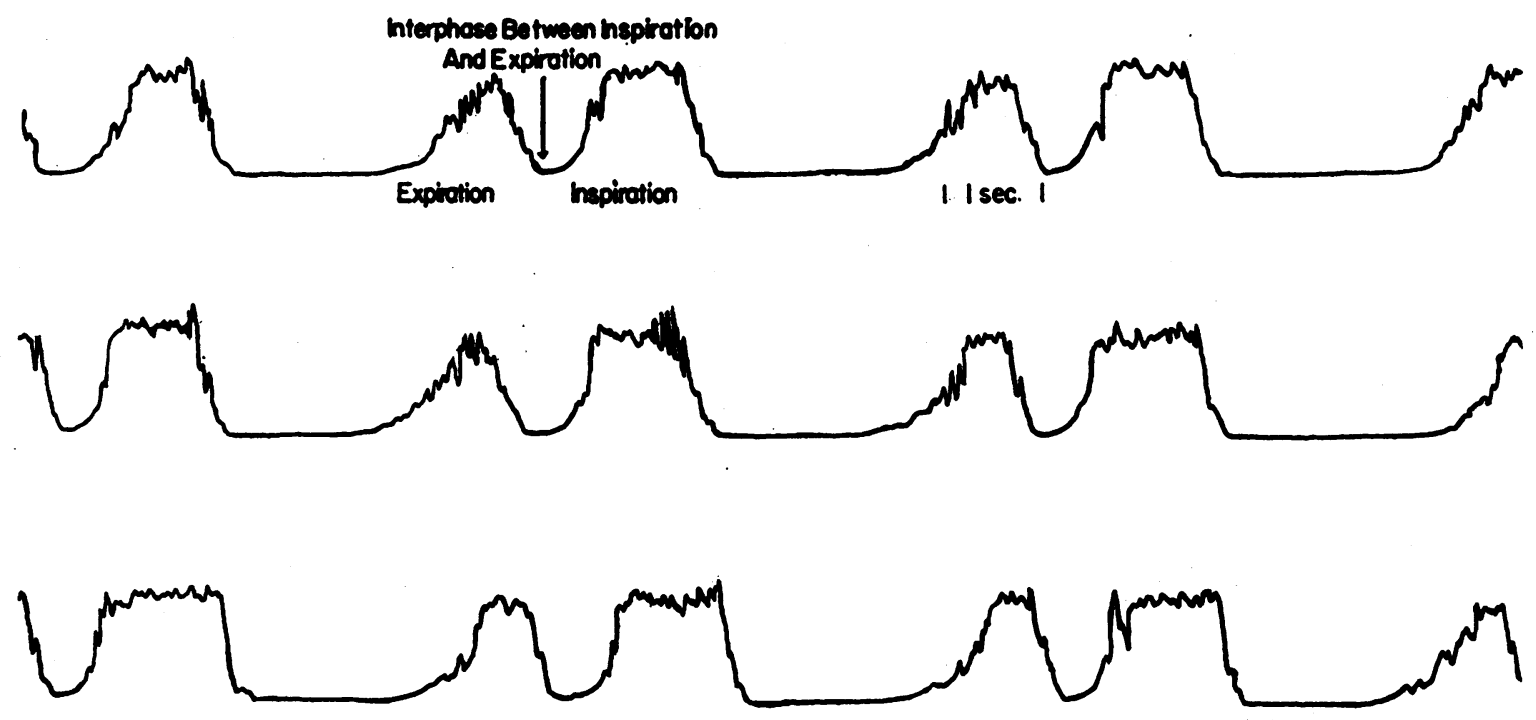

Fig. 3. Acoustic Rispirograx (Read from right to left)

recording as spirometry, pneumography, and airometry. Larval Cheyne-Stokes patterns, periods of relative apnoea, curious types of cogwheel respiration, and marked prolongation of one or the other interphase have been observed. They appear to have significant qualitative and quantitative correlations with the process of falling asleep, with the state of sleep itself, with states of anxiety or of undifferentiated emotional tension, etc. Detailed studies of these physiological and psychological correlations will be reported later (Figure 3).

Furthermore, since the whole moving current of respired air probably contributes to the acoustic energy of the breath sounds, the height of any point on the curve must be related in the first instance to the velocity of a given volume of air passing under the microphone at that instant. It follows, therefore, that theoretically the area subtended by the curve and the base-line must represent one integral of the volume of moving air. For this reason, quantitative volumetric correlations between the acoustic respirogram and the minute respiratory volume may be expected. In Figure 3, however, it is evident that the area subtended by the curve which represents inspiration is not equal to that subtended by the curve which represents expiration. Preliminary experiments with an artificial larynx-trachea-lung have shown that this is because the acoustic energy is influenced also by the direction of motion of the respired air in the respiratory tract. Therefore, separate calibrations are necessary for expiration and inspiration, unless a position can be found for the microphone where a summation occurs of all resonances and of all accompanying acoustic energies. A study aimed to demonstrate quantitative volumetric relations is now under way.

The instrument was developed jointly by one of us (S. M.) and Mr. Paul Traugott of Electro-Physical Laboratories, New York.

This study has been made possible by a grant from the Josiah Macy, Jr. Foundation to the Department of Neurology, of the College of Physicians and Surgeons of Columbia University, New York. The authors are indebted to Drs. Tracy J: Putnam and Paul Hoefer for invaluable encouragement and advice.

\section{BIBLIORAPHY}

1. Kubie, L. S. and Margolin, S., A physiological method for the induction of states of partial sleep, and securing free associations and early memories in such states. Tr. Am. Neurol. A., 1942. (In press.)

2. Kubie, L. S., and Margolin, S., An apparatus for the use of breath sounds as a hypnagogic stimulus. Am. J. Psychiat., 1942-43. (In press.) 